\title{
Opinión del profesorado hacia proyectos colaborativos con Tecnologías de la Información y Comunicación: un estudio psicométrico
}

\author{
Verónica Basilotta Gómez-Pablos ${ }^{1}$ \\ ORCID: 0000-0003-1976-4548 \\ Ana García-Valcárcel Muñoz-Repiso² \\ ORCID: 0000-0003-0463-0192
}

\section{Resumen}

En la escuela el profesorado es cada vez más consciente de la importancia que tiene adaptar la enseñanza y el aprendizaje al entorno y realidad del alumnado, haciendo que estos adquieran destrezas para su día a día y su futuro académico y laboral. Una de las mejores formas es a través del Aprendizaje Basado en Proyectos (ABP), una metodología didáctica que ha tomado auge en las últimas décadas, conectada al desarrollo de competencias que despiertan el interés del alumnado por las tareas académicas. En este contexto, este trabajo tiene como objetivo la construcción de un cuestionario para valorar la opinión del profesorado de centros educativos que participa en proyectos de trabajo colaborativo con TIC (PROCOLTIC). La validación de contenido se ha llevado a cabo a través del acuerdo y consenso de dieciocho expertos. El cuestionario se administró a 310 docentes de diferentes centros educativos de toda España que participan en diversos proyectos. A partir de los resultados obtenidos, se presenta un cuestionario con niveles altos de frabilidad y de validez de constructo. Consideramos que estas características permiten la utilización del cuestionario PROCOLTIC para valorar la percepción de los docentes acerca de los proyectos colaborativos con TIC en los procesos de enseñanza y aprendizaje.

\section{Palabras clave}

TIC - Aprendizaje basado en proyectos - Aprendizaje colaborativo - Instrumento de evaluación - Práctica docente.

1- Universidad a Distancia de Madrid, Madrid, España. Contacto: veronicamagdalena.basilotta@udima.es.

2- Universidad de Salamanca, Salamanca, España. Contacto: anagv@usal.es. 


\section{Teachers' opinion on collaborative projects using Communication and Information Technologies: a psychometric study}

\section{Abstract}

At school, teachers are increasingly aware of the importance of adapting teaching and learning to the students's environment and reality, leading them to earn a series of basic skills for their daily tasks and their academic and professional future. One of the best ways is through Project Based Learning (PBL), a teaching methodology that has caught on in recent decades and is connected to the development of competencies that arouse students' interest in academic tasks. The aim of this work is to construct a questionnaire to evaluate the opinion of teachers in relation to collaborative projects using ICT (PROCOLTIC). The content was validated through the agreement and consensus of 18 experts. The questionnaire was applied to 310 teachers participating in some projects. The results obtained in the validation process generated an opinion scale with high levels of reliability and construct validity. We believe that these technical characteristics allow the use of the PROCOLTIC to evaluate the opinion of teachers on collaborative projects which utilize ICT in teaching and learning.

\section{Keywords}

ICT - Project based learning - Collaborative learning - Assessment instrument - Teaching practice.

\section{Introducción}

En este artículo nos centramos en la valoración de la práctica docente como instrumento de mejora de la calidad del proceso de enseñanza y aprendizaje. Al respecto, Zabala (2002) señala que este análisis debe realizarse a través de los acontecimientos que resultan de la interacción profesor-alumnos y alumnos-alumnos. Para ello es necesario considerar la experiencia del profesorado como una actividad dinámica, reflexiva, que debe abarcar, tanto los procesos de planeación docente, como los de evaluación de los resultados, por ser parte inseparable de su actuación (RUEDA, 2018).

En este sentido, es fundamental que el profesorado evalúe las estrategias, experiencias y herramientas que utiliza para impulsar nuevas formas de aprender y enseñar. Esta evaluación se configura como aliada relevante en la identificación de procesos y prácticas que resulten ser más eficaces. Además, es preciso que identifique y comprenda la potencialidad de las TIC para promover determinadas y necesarias transformaciones en las prácticas educativas. 
En consonancia con lo expuesto entendemos que la evaluación del profesorado tiene una doble finalidad: por una parte, la mejora de los aprendizajes de los estudiantes, y por otra, el perfeccionamiento del propio docente (CABERO; LLORENTE; MORALES, 2018).

Por tanto, las concepciones del profesorado juegan como herramientas que les permiten interpretar la realidad, las cuales no son estáticas, se transforman en la medida en que el profesor y su entorno cambian paulatinamente (GARCÍA; LORED0; CARRANZA, 2008). La reflexión del profesor sobre su quehacer le permite identificar situaciones que puede enfrentar durante su práctica, como las características del grupo, o las inquietudes de los estudiantes, entre otros, que en ocasiones obligan a transformar lo preparado para la clase. Consideramos necesario conocer las concepciones que tienen los docentes de la práctica educativa ya que permiten pensar con fundamento cómo intervenir y mejorar el proceso de enseñanza y aprendizaje (BERTELLE; ITURRALDE; ROCHA, 2003) y en caso de que sea necesario, reorganizar la práctica educativa de acuerdo con los aprendizajes reales y necesidades de los estudiantes.

\section{El aprendizaje basado en proyectos}

Los docentes se enfrentan, cada vez más, a alumnos que tienen una amplia gama de habilidades, que provienen de diversos ámbitos culturales y étnicos, y que aprenden diferentes lenguas e idiomas. Las escuelas, por ello, buscan maneras de responder a las necesidades de los estudiantes. El aprendizaje basado en proyectos (en adelante ABP) es especialmente adecuado para atender la diversidad del aula al incorporar opciones para los diferentes estilos de aprendizaje y niveles curriculares que los estudiantes presentan (BLANCHARD, 2014).

El ABP puede definirse como una estrategia de instrucción real y auténtica, en la que los estudiantes tienen la oportunidad de resolver problemas, tomar decisiones y realizar tareas complejas y desafiantes, que culminan en la elaboración de presentaciones y productos finales. Permite a los estudiantes actuar como pequeños investigadores y les capacita para plantear hipótesis, conocer y experimentar situaciones de la vida real, documentarse, compartir ideas sobre un tema, etc.

Por lo tanto, la base del ABP radica en la autenticidad o la aplicación de la investigación en la vida real. Los estudiantes reciben una pregunta guía o driving question que deben resolver, y luego se les pide que creen un producto para presentar el conocimiento y aprendizajes adquiridos durante el proceso (THAMARASSERI, 2014).

Es evidente que una educación para la diversidad ha de basarse necesariamente en una concepción curricular amplia, que ofrezca oportunidades a todos los estudiantes para situarse dentro del currículum escolar regular (OJEA, 2000). El ABP puede constituir una alternativa a esta globalidad en cuanto se caracteriza por 1) la relevancia de la visión constructivista sobre el aprendizaje; 2) la importancia que se le otorga al contexto de aprendizaje; y 3) el papel de las estrategias metacognitivas como forma de pensar sobre el proceso de planificación, organización e investigación de la información (LACUEVA, 2016).

De esta forma, el ABP puede ser clave para conseguir una educación integral, que favorezca el desarrollo de la afectividad, la clarificación y aplicación de positivos valores, 
la prudencia y pertinencia práxicas y las capacidades metacognitivas. Se configura como una estrategia que promueve la participación activa en el aula, en un clima de confianza y respeto, y que impulsa la formación de ciudadanos críticos, conocedores de su realidad.

Blanchard (2014) define el ABP como una metodología globalizadora que relaciona tres elementos fundamentales para lograr un aprendizaje significativo y aplicado a la realidad: por un lado, los intereses de los estudiantes, por otro el currículo establecido y, junto a estos, las necesidades del contexto en el que se desarrolla la práctica educativa. El docente como mediador deberá unir la curiosidad investigadora del alumnado, con la sistematización de los aprendizajes planteados desde el currículo oficial.

El tipo de proyecto que posee una máxima potencialidad educativa es aquel que se lleva a cabo mediante el trabajo colaborativo del alumnado en el aula. Esta dinámica provoca que nadie por sí solo pueda alcanzar la meta si sus compañeros no lo hacen. Este tipo de interacción implica actitudes facilitadoras de ayuda, y los estudiantes son estimulados tanto por sus propios compañeros como por los profesores, lo que repercute sobre la motivación y finalmente en el rendimiento académico (ANDREU, 2016; JOHNSON; JOHNSON, 2009).

Si revisamos las investigaciones que aluden al ABP en la escuela, y su repercusión en el aprendizaje del alumnado, encontramos que son numerosas las aportaciones que se exponen. Thomas (2000) y Condliffe y otros autores(2017) encontraron evidencias de que el ABP puede apoyar el aprendizaje del estudiante y ser más eficaz que los métodos de instrucción tradicionales.

En esta misma línea, son varios los autores que han encontrado asociaciones positivas entre el ABP y el desarrollo de conocimientos, habilidades cognitivas y sociales en los estudiantes (FOGLEMAN; MCNEILL; KRAJCIK, 2011; HALVORSEN et al., 2012; HARRIS et al., 2014; PARKER et al., 2011). Estos estudios muestran que si el ABP se aplica de una manera adecuada en el aula los estudiantes pueden retener más conocimientos y mejorar sus actitudes con respecto al aprendizaje, desarrollar su capacidad creativa, trabajar en equipo, y adquirir un gran manejo de la tecnología (VEGA, 2015).

Sin embargo, existe un número significativamente menor de estudios que abordan las opiniones de los profesores sobre la eficacia de esta metodología (ENGLISH, 2013; LACUEVA, 2015). En este contexto, hemos encontrado el estudio de Cyprian (2014) que se centró en investigar la autoeficacia de seis profesores que implementaron el ABP en una escuela en el suroeste de Estados Unidos. La investigación reveló que influyeron varios factores en la autoeficacia de los docentes cuando implementaban el ABP. Los factores incluyeron el contenido de la asignatura, el entorno de la clase, la autoestima de los profesores y su formación.

El estudio de Habok y Nagy (2016) con 109 docentes de escuelas primarias y secundarias reveló numerosas diferencias entre sus opiniones basadas en el grado de experiencia y el tipo de escuela. En general, los docentes preferían la metodología de $\mathrm{ABP}$ a la tradicional, se autopercibían como facilitadores del proceso, y consideraban la motivación y la transmisión de valores como parte central del trabajo del proyecto.

En este contexto, y en base a la escasez de instrumentos de medida válidos y fiables (THOMAS, 2000), en este ámbito, consideramos conveniente diseñar un cuestionario sobre el ABP, desde la perspectiva del profesorado. Pensamos que es necesario profundizar en la evaluación de los proyectos que se desarrollan desde esta perspectiva del aprendizaje 
para garantizar la efectividad y calidad de estas estrategias puestas en marcha en el marco escolar. Es un hecho que la opinión del profesorado tiene una importancia crítica para el éxito de esta metodología. Así como opinamos que los docentes pueden ofrecer una información valiosa para analizar su experiencia de aprendizaje y evaluar si los proyectos se están desarrollando de forma adecuada.

\section{Objetivos}

Los objetivos de este trabajo de investigación se concretan en los siguientes: 1) Construir y validar un cuestionario-escala para evaluar la percepción del profesorado frente a los proyectos de trabajo colaborativo con TIC (PROCOLTIC); 2) Realizar un acercamiento a la opinión del profesorado implicado en proyectos de trabajo colaborativo con TIC en centros educativos en España.

\section{Método}

Para recoger las valoraciones del profesorado acerca de los proyectos colaborativos con TIC, se ha diseñado un cuestionario con preguntas tipo likert, con un rango de valoración de 0 a 4 . Se ha elegido este instrumento y no otro, principalmente por el ahorro que supone para los costes de la investigación y porque es posible llegar a más sujetos residentes en zonas diferentes sin necesidad de desplazarse. Asimismo se pueden señalar otros motivos que justifican esta decisión (CORBETTA, 2010):

- ofrece mayor flexibilidad al entrevistado, que puede cumplimentar el cuestionario cuando mejor le convenga;

- mayor garantía de anonimato en comparación con la entrevista cara a cara, dado que el cuestionario cumplimentado no debe contener elementos que permitan identificar al sujeto que lo ha realizado;

- ausencia de distorsiones debidas al entrevistador.

Según Del Rincón y otros autores (1995) un cuestionario es uno de los instrumentos de uso más generalizado en el campo de la investigación social, destacando en su empleo la posibilidad de estimar magnitudes absolutas o relativas a variables en una población determinada. Por ello, la decisión de emplear el cuestionario en el estudio se deriva, además de las consideraciones de diferentes autores consultados (ARNAL; DEL RINCÓN; LATORRE, 1992; DEL RINCÓN, 1995; MCMILLAN; SCHUMARCHER, 2005), de la posibilidad de realizarlo en formato electrónico, facilitando en gran medida tanto su distribución como su recogida.

\section{Proceso de elaboración del cuestionario-escala PROCOLTIC}

Antes de elaborar el cuestionario realizamos un proceso de reflexión sobre el tipo de información que deseábamos obtener y, por consiguiente, sobre el tipo de preguntas y el modo de plantearlas para que los docentes las pudieran contestar sin dificultades de 
interpretación. Para la redacción de los ítems se siguieron las recomendaciones generales de autores como Morales, Urosa y Blanco (2003): ítems redactados con claridad, es decir, que expresen directamente el aspecto que se desea evaluar y que contengan una única idea. Además, todas las cuestiones deben estar relacionadas con el tema de estudio, los ítems se deben plantear de manera sencilla, concisa, clara y sin ningún tipo de ambigüedad.

En base a estas recomendaciones, se elaboró una primera versión experimental de 40 ítems con 5 categorías de respuesta distribuidos en seis dimensiones, identificadas a partir de las aportaciones teóricas de diferentes autores (BRUNDIERS; WIEK, 2013; DU; HAN, 2016; ERTMER; SIMONS, 2006; INTYKBEKOV, 2017; JOHNSON; JOHNSON, 2009; MADOYAN, 2016; MERGENDOLLER; THOMAS, 2000): contextualización del centro, características del proyecto, papel del profesorado, herramientas utilizadas, papel del alumnado y resultados obtenidos. Las dimensiones se definen como sigue:

a. Contextualización del centro: esta primera dimensión aparece como fundamental en la literatura, ya que es esencial conocer con qué infraestructura y recursos tecnológicos cuenta el centro educativo dónde se desarrolla el proyecto, así como algunos aspectos relacionados con la organización del centro; para saber si influye o no en la realización de estas prácticas educativas.

b. Características del proyecto: otra de las dimensiones identificadas como relevantes es la opinión del profesorado acerca de las características del proyecto en el que participa. Para ello, se pretende recabar su opinión sobre la planificación y organización del proyecto, los elementos curriculares, la distribución de las tareas, las tecnologías utilizadas y la implicación del alumnado (DU; HAN, 2016).

c. Papel del profesorado: el papel del profesorado en los proyectos merece una reflexión especial y es que se trata de una figura clave en el desarrollo y seguimiento del proyecto, que tratará de dinamizar las actividades, animar y motivar a sus alumnos en la realización de las mismas (BRUNDIERS; WIEK, 2013; ERTMER; SIMONS, 2006; INTYKBEKOV, 2017; MADOYAN, 2016).

d. Herramientas utilizadas: se considera que esta dimensión es importante, ya que interesa conocer, por un lado, qué tipos de herramientas tecnológicas se han utilizado en el transcurso del proyecto para fomentar la participación y colaboración del alumnado y del profesorado, así como la difusión del proyecto; $\mathrm{y}$, por otro lado, si el profesorado ha elaborado sus propios materiales y ha utilizado diferentes estrategias e instrumentos para valorar el trabajo del alumnado (ALBA; CARBALLO, 2005). En la elaboración de los ítems de esta dimensión se ha seguido como referencia la clasificación de herramientas de la Web 2.0 que utiliza Bernal (2009).

e. Papel del alumnado: otra de las dimensiones que aparecen como determinante en los estudios revisados, es el papel que se le asigna en todo este proceso al alumnado como protagonista de su aprendizaje. Por ello, y siguiendo como referencia a Johnson y Johnson (2009), se pretende preguntar al profesorado sobre el desempeño y consecución de algunas características del aprendizaje colaborativo por parte del alumnado.

f. Resultados obtenidos: según la teoría revisada, el trabajo por proyectos constituye un método muy eficaz para fortalecer, enriquecer y desarrollar las competencias 
del profesorado. El profesorado puede realizar su labor de una manera más dinámica e interactiva, logrando desarrollar competencias más técnicas por el empleo de las herramientas tecnológicas, así como competencias sociales y pedagógicas. Por ello, se identifica esta dimensión para conocer la opinión del profesorado en relación a los resultados conseguidos en términos de competencias (MERGENDOLLER; THOMAS, 2000).

A continuación, determinamos la forma en que el cuestionario debía aparecer ante los docentes. Se ha tenido en cuenta el formato y el tipo de letra de manera que contribuyan a la comprensión del texto.

La validez de contenido se garantiza a través de jueces expertos. La primera versión del instrumento se sometió, en primer lugar, al análisis y discusión de algunos miembros del grupo de investigación que participan en este estudio, donde se realizaron determinadas correcciones a partir de los criterios cualitativos que obtuvieron mayor consenso. Una vez modificado el cuestionario se sometió el mismo a una segunda ronda de consulta a un grupo de expertos seleccionados, con la intención de recabar los criterios cuantitativos y cualitativos más estables (GARCÍA VALCÁRCEL; BASILOTTA, 2017).

La selección del grupo de expertos se realizó tomando como criterio fundamental de selección la competencia de los candidatos en la temática de la investigación. Se seleccionaron dieciocho personas, entre ellos profesores universitarios y docentes de centros educativos con experiencia en ABP.

El envío y la recepción del cuestionario se realizó por correo electrónico en archivo adjunto, seguido del correspondiente instrumento de validación. Este último se estructuró en dos partes (GARCÍA; CABERO, 2011): la primera parte recogía un texto con la finalidad de agradecer al profesorado su colaboración e informarle del proceso, además de darle algunas orientaciones sobre el proceso de validación (presentación e instrucciones para el proceso de respuesta).

La segunda parte consultaba la idoneidad de los ítems, la cual se ejerció por una doble vía: la cuantitativa, mediante la que empleando una escala de respuesta tipo Likert con 3 categorías evaluaba la relevancia o el grado en el que el ítem es importante para valorar la dimensión que representa, y la claridad o el grado en el que el ítem se comprende fácilmente; la cualitativa, que se establece mediante un conjunto de preguntas abiertas relacionadas con la eliminación, la modificación o la incorporación de ítems; aparte de cualquier otro aspecto que considerasen oportuno.

Los datos aportados por los revisores fueron analizados de acuerdo a su naturaleza. Así, se siguió una metodología cuantitativa para seleccionar los ítems en función de los resultados obtenidos en los criterios de relevancia y claridad. Por otra parte, se utilizó una metodología cualitativa con el objeto de analizar el contenido de las cuestiones abiertas. En base a la información aportada por los revisores se llevaron a cabo las modificaciones oportunas. De los cuarenta ítems que integraban el cuestionario, catorce de ellos no sufren modificación alguna, ya que los expertos no proponen otra versión. Tres ítems son eliminados. Por un lado, los jueces consideran que el ítem Ha dado instrucciones claras a los alumnos para realizar las tareas no es relevante para este tipo de autoevaluación. Por otra parte, el item El proyecto promueve el desarrollo profesional del docente puede 
sobrentenderse con el ítem El proyecto es una buena oportunidad para la formación del profesorado. El tercer ítem Los programas o plataformas utilizadas en el proyecto son eficaces se elimina porque los jueces consideran que es similar al item Las tecnologías utilizadas en el proyecto facilitan su adecuada realización.

También se añaden tres nuevos ítems siguiendo las recomendaciones de los expertos, que destacan la necesidad de analizar algunos de los elementos del aprendizaje colaborativo: interacción, reflexión, interdependencia positiva y el desarrollo de habilidades sociales en el alumnado (El alumnado ha tenido suficientes oportunidades para interactuar $y$ colaborar con sus compañeros; El alumnado ha tenido (tiene) tiempo para reflexionar conjuntamente sobre el proceso de trabajo realizado; El alumnado ha asumido (asume) roles complementarios para el desempeño de las tareas).

A su vez, se añaden dos items sobre competencia pedagógica y competencia comunicativa/social en el profesorado (El proyecto le ha permitido aprender-adquirir competencias pedagógicas para la docencia; El proyecto le ha permitido aprender-adquirir competencias sociales para la docencia (compañerismo, trabajo en equipo, respeto, etc.). Además los jueces sugieren incorporar un ítem que valore si los proyectos suponen una oportunidad para un cambio en la cultura de los centros educativos.

Por último, veintitrés ítems son modificados de acuerdo a la opinión de los jueces, que, en general, atienden a la siguiente tipología: cambios ortográficos y de puntuación, cambios sintácticos y cambios en el orden de algunos ítems.

\section{Población y muestra}

La población está constituida por el conjunto de profesores que participaron en proyectos de trabajo colaborativo con TIC en el curso académico 2014/2015 en diversos centros educativos en España. En el curso 2014/2015 se registraron un total de 83 proyectos colaborativos con TIC en los cuales participaron 553 profesores, distribuidos de la siguiente manera: 241 hombres (43.4\%) y 312 mujeres (56.6\%). Por lo tanto, hemos partido de una población de $\mathrm{N}=553$ para calcular el tamaño muestral. Así, mediante la aplicación de la correspondiente fórmula (ARNAL; DEL RINCÓN; LATORRE, 1992),

$$
\mathrm{n}=\frac{Z_{\alpha}^{2} p q N}{e^{2}(N-1)+Z_{\alpha}^{2} p q}
$$

con un nivel de homogeneidad máximo ( $\mathrm{p}=\mathrm{q}=0.5)$, un nivel de confianza del $96 \%(\mathrm{Z}=2.05)$ y un error muestral (e) del 4\%, el tamaño muestral recomendado es de 300 sujetos. Después de solicitar por twitter y correo electrónico la colaboración de los docentes, gracias a la tecnología de Encuestafacil mediante un cuestionario electrónico; se recogieron en 2015, 310 cuestionarios, estableciendo éste como tamaño final de la muestra. En la Tabla 1 se presenta la distribución de la población y la muestra según el nivel educativo en el que imparte docencia el profesorado. 
Tabla 1- Muestra de profesores por nivel educativo. Representatividad.

\begin{tabular}{|c|c|c|c|c|}
\hline \multirow[b]{2}{*}{ Nivel educativo } & \multicolumn{2}{|c|}{ Población } & \multicolumn{2}{|c|}{ Muestra } \\
\hline & $\mathrm{F}$ & $\%$ & $F$ & $\%$ \\
\hline Educación Infantil & 57 & 10.30 & 28 & 9.0 \\
\hline Educación Primaria & 245 & 44.30 & 147 & 47.4 \\
\hline Educación Secundaria & 178 & 32.18 & 97 & 31.3 \\
\hline Bachillerato & 41 & 7.41 & 23 & 7.4 \\
\hline Formación Profesional & 32 & 5.78 & 15 & 4.8 \\
\hline Total & 553 & 100 & 310 & 100 \\
\hline
\end{tabular}

Fuente: Elaboración propia.

\section{Análisis de los datos}

Los datos cualitativos han sido analizados mediante análisis de contenido. Respecto a los datos cuantitativos, el cálculo de los estadísticos descriptivos, el análisis factorial exploratorio, así como la estimación de la consistencia interna; se han realizado con el programa SPSS Statistics 23.0.

\section{Resultados}

\section{Validación del cuestionario}

\section{Fiabilidad}

Para este análisis se utilizó la prueba $\alpha$ de Cronbach, obteniéndose un valor de 0.922 . De modo general, parece que la escala presenta un índice de fiabilidad muy elevado, sin embargo, se pretende depurar la escala reduciendo el número de ítems. En la Tabla 2 se puede ver el resultado de dicho índice de consistencia interna para el cuestionario de manera general y para cada uno de los diferentes constructos establecidos. El alfa de Cronbach se sitúa en todos los casos por encima de 0.70 a excepción de la dimensión papel del profesorado que se sitúa en 0.661 .

Tabla 2 - $\alpha$ de Cronbach para las sub-escalas. Cuestionario PROCOLTIC

\begin{tabular}{|c|c|c|c|}
\hline & $\boldsymbol{\alpha}$ de Cronbach & $\boldsymbol{\alpha}$ de Cronbach basado en los elementos tip. & $N$ de elementos \\
\hline Cuestionario completo & .922 & .936 & 43 \\
\hline A. Contextualización del centro & .760 & .761 & 6 \\
\hline B. Características del proyecto & .851 & .864 & 8 \\
\hline C. Papel del profesorado & .661 & .729 & 7 \\
\hline D. Herramientas utilizadas & .789 & .793 & 5 \\
\hline E. Papel del alumnado & .901 & .902 & 11 \\
\hline F. Resultados obtenidos & .849 & .849 & 6 \\
\hline
\end{tabular}

Fuente: Elaboración propia. 
La Tabla 3 nos muestra unos índices de correlación ítem-total no aceptables en el ítem 4, Se ha sentido (se siente) apoyado por los compañeros docentes en el desarrollo del proyecto, le han proporcionado ayuda y asesoramiento. Analizando el contenido del ítem, se decide eliminarlo. Tras la eliminación de este ítem por su baja relación, la dimensión pasa a tener seis ítems y un índice de consistencia interna de $\alpha$ de Cronbach de .715 frente al anterior de .661 con 7 ítems.

Tabla 3 - Estadísticos total-elemento. Dimensión papel del profesorado

\begin{tabular}{|c|c|c|c|c|c|}
\hline Estadísticos total-elemento & $\begin{array}{l}\text { Media sin el } \\
\text { elemento }\end{array}$ & $\begin{array}{l}\text { Varianza sin } \\
\text { el elemento }\end{array}$ & $\begin{array}{l}\text { Correlación } \\
\text { elemento-total } \\
\text { corregida }\end{array}$ & $\begin{array}{c}\text { Correlación } \\
\text { múltiple al } \\
\text { cuadrado }\end{array}$ & $\begin{array}{l}\alpha \text { de } \\
\text { Cronbach sin } \\
\text { el elemento }\end{array}$ \\
\hline $\begin{array}{l}\text { C1. Ha participado (participa) activamente en la } \\
\text { planificación y diseño del proyecto. }\end{array}$ & 20.67 & 7.122 & .382 & .427 & .649 \\
\hline $\begin{array}{l}\text { C2. Ha participado (participa) activamente en el } \\
\text { desarrollo del proyecto. }\end{array}$ & 20.16 & 8.209 & .534 & .497 & .576 \\
\hline $\begin{array}{l}\text { C3. Ha tenido (tiene) una actitud favorable durante el } \\
\text { desarrollo del proyecto. }\end{array}$ & 19.85 & 9.760 & .490 & .301 & .617 \\
\hline $\begin{array}{l}\text { C4. Se ha sentido (se siente) apoyado por los } \\
\text { compañeros docentes en el desarrollo del proyecto: le } \\
\text { han proporcionado ayuda y asesoramiento. }\end{array}$ & 20.58 & 9.674 & .118 & .082 & .715 \\
\hline $\begin{array}{l}\text { C5. Se ha sentido (se siente) cómodo empleando las } \\
\text { herramientas tecnológicas utilizadas en el proyecto } \\
\text { colaborativo. }\end{array}$ & 19.96 & 9.704 & .428 & .280 & .623 \\
\hline $\begin{array}{l}\text { C6. Ha animado (anima) al alumnado más reticente a } \\
\text { realizar las actividades del proyecto. }\end{array}$ & 19.96 & 9.212 & .520 & .432 & .600 \\
\hline $\begin{array}{l}\text { C7. Ha llevado (lleva) a cabo un seguimiento y registro } \\
\text { del trabajo realizado por el alumnado durante el proyecto. }\end{array}$ & 20.26 & 8.768 & .476 & .374 & .598 \\
\hline
\end{tabular}

Fuente: Elaboración propia.

Tras los cambios realizados, el cuestionario de manera global concluye con 42 items y un índice de consistencia interna de $\alpha$ de Cronbach de .923. Los estadísticos obtenidos para el $\alpha$ de Cronbach en todas las dimensiones muestran valores por encima de 0.70 . Por lo tanto, y teniendo en cuenta que el valor mínimo de $\alpha$ de Cronbach lo podemos situar en torno a 0.70 (Morales et al., 2003), estamos en condiciones de afirmar que las dimensiones tienen fiabilidades satisfactorias. Esto quiere decir que los ítems propuestos son precisos en su medición, y que miden adecuadamente los constructos. 


\section{Validez de contenido}

En cuanto a la validez de contenido, se remite al lector al apartado de elaboración del cuestionario (Método), en el que se muestra el proceso de validación por jueces o expertos para establecer los ítems que conforman el instrumento. Por otra parte, el propio equipo investigador actuó desde su rol de experto para analizar la relevancia y claridad de los ítems con respecto a los presupuestos teóricos y su relación con las dimensiones identificadas.

\section{Validez de constructo}

El estudio de la estructura factorial del cuestionario se realizó a través de un análisis factorial de tipo exploratorio usando Componentes principales como método de extracción. Como el análisis de los componentes principales no exige los supuestos de normalidad, se procede a la aplicación directa sobre todos los ítems forzando la extracción de 6 factores. La idoneidad de realizar el análisis factorial se confirmó calculando el índice de Kaiser-Meyer-Olkin y la prueba de esfericidad de Bartlett $(\mathrm{KMO}=.873 ;$ Chi-cuadrado $=6282.098 ; \mathrm{gl}=861 ; \mathrm{p}<.001)$. Dada la interrelación entre los factores $(\mathrm{r}=.341)$, se utilizó Oblimin como método de rotación, la cual supone que los factores están asociados entre sí. Se exploró el análisis especificando un valor delta igual a cero. Los resultados se presentan en la Tabla 4, donde se puede observar que las comunalidades de los ítems que componen la escala están todas por encima de .30. En la Tabla 4 hemos resaltado las correlaciones ítem-elemento superiores a .40 (MORALES, 2006).

La matriz de estructura (método oblimin), como se puede observar en la Tabla 4, sugiere que los supuestos teóricos iniciales sobre la dimensionalidad del cuestionario pueden ser acertados. La solución factorial converge en once iteraciones y explica en conjunto el 53.52\% de la varianza. En la estructura factorial resultante (Tabla 5), nos encontramos un primer factor con un valor de $\lambda=10.634$ que explica por sí solo el $25.32 \%$ de la variabilidad asociada a la matriz de correlaciones, definido por saturaciones en él de ocho ítems de la escala. El segundo factor con un valor de $\lambda=3.014$ que explica por sí solo el $7.177 \%$ de la variabilidad, el tercer factor con un valor de $\lambda=2.725$ que explica por sí solo el $6.488 \%$ de la varianza, el cuarto factor con un valor de $\lambda=2.357$ que explica el 5.611\% de la varianza, el quinto factor con un valor de $\lambda=2.039$ que explica el $4.856 \%$ de la varianza, y el sexto factor con un valor $\lambda=1.709$ que explica el $4.069 \%$ de la varianza.

En la Tabla 6 se recoge la correlación entre los factores obtenidos en el análisis factorial exploratorio. El valor de los coeficientes indica que los factores poseen información común, por lo que el método de rotación empleado es el más apropiado en este caso (OLMOS et al., 2014). 
Verónica Basilotta GÓMEZ-PABLOS; Ana García-Valcárcel MUÑOZ-REPISO

Tabla 4 - Saturación de los ítems en los factores

\begin{tabular}{|c|c|c|c|c|c|c|c|c|}
\hline \multicolumn{9}{|c|}{ Matriz de estructura } \\
\hline \multicolumn{9}{|c|}{ Componente } \\
\hline & & 1 & 2 & 3 & 4 & 5 & 6 & Comunalidad \\
\hline \multirow{6}{*}{ A. Contextualización del centro } & 11 & & & .791 & & & & .653 \\
\hline & 12 & & & .750 & & & & .627 \\
\hline & 13 & & & .715 & & & & .532 \\
\hline & 14 & & & .538 & & & & .463 \\
\hline & 15 & & & .633 & & & & .442 \\
\hline & 16 & & & .570 & & & & .369 \\
\hline \multirow{8}{*}{ B. Características del proyecto } & 17 & .631 & & & & & & .462 \\
\hline & 18 & .797 & & & & & & .648 \\
\hline & 19 & .726 & & & & & & .564 \\
\hline & 110 & .706 & & & & & & .507 \\
\hline & 111 & .778 & & & & & & .612 \\
\hline & 112 & .713 & & & & & & .547 \\
\hline & 113 & .662 & & & & & & .501 \\
\hline & 114 & .634 & & & & & & .457 \\
\hline \multirow{6}{*}{ C. Papel del profesorado } & 115 & & & & & & .792 & .644 \\
\hline & 116 & & & & & & .839 & .721 \\
\hline & 117 & & & & & & .542 & .447 \\
\hline & 118 & & & & & & .407 & .358 \\
\hline & 119 & & & & & & .507 & .453 \\
\hline & 120 & & & & & & .497 & .489 \\
\hline \multirow{5}{*}{ D. Herramientas utilizadas } & 121 & & & & .620 & & & .398 \\
\hline & 122 & & & & .811 & & & .675 \\
\hline & 123 & & & & .754 & & & .582 \\
\hline & 124 & & & & .702 & & & .504 \\
\hline & 125 & & & & .683 & & & .498 \\
\hline \multirow{11}{*}{ E. Papel del alumnado } & 126 & & -.747 & & & & & .561 \\
\hline & 127 & & -.670 & & & & & .465 \\
\hline & 128 & & -.700 & & & & & .520 \\
\hline & 129 & & -.680 & & & & & .486 \\
\hline & 130 & & -.690 & & & & & .491 \\
\hline & 131 & & -.775 & & & & & .615 \\
\hline & 132 & & -.749 & & & & & .563 \\
\hline & 133 & & -.650 & & & & & .461 \\
\hline & 134 & & -.720 & & & & & .563 \\
\hline & 135 & & -.703 & & & & & .526 \\
\hline & 136 & & -.698 & & & & & .534 \\
\hline \multirow{6}{*}{ F. Resultados obtenidos } & 137 & & & & & .778 & & .631 \\
\hline & 138 & & & & & .789 & & .651 \\
\hline & 139 & & & & & .762 & & .615 \\
\hline & 140 & & & & & .692 & & .598 \\
\hline & 141 & & & & & .766 & & .596 \\
\hline & 142 & & & & & .633 & & .448 \\
\hline
\end{tabular}

Método de extracción: análisis de componentes principales.

Método de rotación: Oblimin con normalización Kaiser

Fuente: Elaboración propia. 
Tabla 5 - Solución factorial de seis factores, con los valores y el porcentaje de varianza explicado por cada uno de ellos

\begin{tabular}{c|c|c|c}
\hline \multirow{2}{*}{ Factores } & \multicolumn{3}{c}{ Sumas de extracción de cargas al cuadrado } \\
\cline { 2 - 4 } & Total & \% de varianza & \% acumulado \\
\hline 1 & 10.634 & 25.320 & 25.320 \\
\hline 2 & 3.014 & 7.177 & 32.497 \\
\hline 3 & 2.725 & 6.488 & 38.984 \\
\hline 4 & 2.357 & 5.611 & 44.596 \\
\hline 5 & 2.039 & 4.856 & 49.451 \\
\hline 6 & 1.709 & 4.069 & 53.521 \\
\hline
\end{tabular}

Fuente: Elaboración propia.

Tabla 6 - Matriz de correlaciones entre factores

\begin{tabular}{|c|c|c|c|c|c|c|}
\hline Factores & 1 & 2 & 3 & 4 & 5 & 6 \\
\hline 1 & 1.000 & -.341 & .156 & .252 & .344 & .221 \\
\hline 2 & -.341 & 1.000 & -.161 & -.290 & -.293 & -.219 \\
\hline 3 & .156 & -.161 & 1.000 & .062 & .075 & .064 \\
\hline 4 & .252 & -.290 & .062 & 1.000 & .192 & .166 \\
\hline 5 & .344 & -.293 & .075 & .192 & 1.000 & .116 \\
\hline 6 & .221 & -.219 & .064 & .166 & .116 & 1.000 \\
\hline
\end{tabular}

Fuente: Elaboración propia.

\section{Acercamiento a la opinión del profesorado implicado en proyectos de trabajo colaborativo con TIC en centros educativos de toda España}

Tras administrar el cuestionario piloto pasamos a analizar la muestra. El cuestionario fue respondido por 310 docentes. En primera instancia se realizaron descriptivos univariados de todos los ítems que nos dieran una primera información sobre las opiniones que presentaba el profesorado en cuestión. Así, en términos generales, los resultados son siempre elevados.

La excepción a las valoraciones elevadas la muestra los primeros seis ítems sobre la idoneidad de las características contextuales del centro, de la que se desprende que el profesorado opina que dichas características, en algunos casos, dificulta la puesta en práctica de los proyectos en los centros. Así, el 33.2\% del profesorado no juzga positivamente la actitud del equipo directivo del centro para apoyar e impulsar el desarrollo del aprendizaje por proyectos, el 34.2\% opina que las características del centro/aula no son apropiadas/ adecuadas para el desarrollo de proyectos colaborativos con TIC (instalaciones, espacios, etc.) y el 52.5\% del profesorado rechaza la idea de que el centro disponga de una velocidad de conexión a Internet adecuada. 
En cuanto a la dimensión relacionada con el papel del profesorado, la mayoría afirma haber participado activamente en el desarrollo del proyecto (83.2\%), haber animado al alumnado más reticente a realizar las tareas y actividades (94.9\%), así como llevar a cabo el seguimiento y registro del trabajo realizado por el alumnado (85.8\%). Esta última cuestión es determinante en el diseño de actividades colaborativas, que requieren de un esfuerzo de planificación y seguimiento importante.

En estos procesos, los docentes afirman haber utilizado diferentes recursos tecnológicos y aplicaciones de la Web 2.0, especialmente aquellas que facilitan la creación y publicación de contenidos, como blogs, wikis, webquest, podcast y ofimática colaborativa (89.4\%).

Si examinamos en profundidad la dimensión papel del alumnado, el valor de la media se distribuye, en la mayoría de los ítems, por encima de 3, esto indica una propensión a la aceptabilidad de las cuestiones analizadas. La media más baja la presenta el ítem 29 El alumnado ha tenido (tiene) tiempo para reflexionar conjuntamente sobre el proceso de trabajo realizado, en cuyo caso se sitúa por debajo de 3, a pesar de que un 73\% de los docentes están de acuerdo con esta afirmación.

Si continuamos analizando los ítems de esta dimensión, observamos que la moda se establece, en algunas ocasiones, en la indiferencia o punto medio de la escala (2), concretamente en el ítem 31 El alumnado se ha sentido apoyado por sus compañeros en el desarrollo del proyecto, le han proporcionado ayuda, ánimo y refuerzo; y en el ítem 32 El alumnado ha ayudado a los compañeros con dificultades para realizar las tareas. Este resultado evidencia que en general, los docentes dudan sobre el hecho de que los estudiantes se ayuden entre ellos en la realización de las tareas del proyecto.

\section{Discusión y conclusiones}

Los resultados obtenidos muestran que, en general, el cuestionario PROCOLTIC tiene una calidad métrica satisfactoria. En concreto, el análisis de la fiabilidad, utilizando el indice Alfa de Cronbach, permite señalar que el cuestionario que presentamos en este estudio, aplicado a una amplia muestra de docentes, posee alta consistencia interna, lo que facilita su aplicación en diferentes contextos (ORGANISTA et al., 2017; SALCINES; GONZÁLEZ, 2016).

En cuanto al análisis de constructo, el análisis presenta unos índices KMO de adecuación muestral y esfericidad significativos. Además los resultados obtenidos sugieren que la escala presentada mide adecuadamente la percepción del profesorado en las dimensiones identificadas, como son la contextualización del centro, características del proyecto, papel del profesorado, herramientas utilizadas, papel del alumnado y resultados obtenidos. 
Opinión del profesorado hacia proyectos colaborativos con Tecnologías de la Información y Comunicación: un...

Tabla 7 - Puntuación media en cada opción de la escala, desviación típica y correlación ítem-total

\begin{tabular}{|c|c|c|c|c|}
\hline & Ítems & $\mathrm{N}$ & Media & Sx \\
\hline \multirow{6}{*}{ A. Contextualización del centro } & 11 & 310 & 2.66 & 1.072 \\
\hline & 12 & 310 & 2.79 & 1.062 \\
\hline & 13 & 310 & 2.18 & 1.247 \\
\hline & 14 & 310 & 2.85 & 1.032 \\
\hline & 15 & 310 & 2.49 & 1.070 \\
\hline & 16 & 310 & 2.80 & 1.094 \\
\hline \multirow{8}{*}{ B. Características del proyecto } & 17 & 310 & 3.26 & .816 \\
\hline & 18 & 310 & 3.33 & .752 \\
\hline & 19 & 310 & 3.36 & .758 \\
\hline & 110 & 310 & 3.57 & .575 \\
\hline & 111 & 310 & 3.27 & .699 \\
\hline & 112 & 310 & 3.41 & .699 \\
\hline & 113 & 310 & 3.49 & .627 \\
\hline & 114 & 310 & 3.58 & .579 \\
\hline \multirow{6}{*}{ C. Papel del profesorado } & 115 & 310 & 2.91 & 1.308 \\
\hline & I16 & 310 & 3.41 & .842 \\
\hline & 117 & 310 & 3.73 & .488 \\
\hline & 118 & 310 & 3.62 & .555 \\
\hline & 119 & 310 & 3.61 & .607 \\
\hline & 120 & 310 & 3.32 & .761 \\
\hline \multirow{5}{*}{ D. Herramientas utilizadas } & 121 & 310 & 3.08 & .859 \\
\hline & 122 & 310 & 3.28 & .832 \\
\hline & 123 & 310 & 3.47 & .779 \\
\hline & 124 & 310 & 3.10 & .983 \\
\hline & 125 & 310 & 3.02 & .921 \\
\hline \multirow{11}{*}{ E. Papel del alumnado } & 126 & 310 & 3.23 & .625 \\
\hline & 127 & 310 & 3.05 & .715 \\
\hline & 128 & 310 & 3.26 & .733 \\
\hline & 129 & 310 & 2.80 & .730 \\
\hline & 130 & 310 & 3.17 & .731 \\
\hline & 131 & 310 & 2.90 & .832 \\
\hline & 132 & 310 & 2.91 & .859 \\
\hline & 133 & 310 & 3.15 & .640 \\
\hline & 134 & 310 & 3.32 & .642 \\
\hline & 135 & 310 & 3.29 & .629 \\
\hline & 136 & 310 & 3.33 & .646 \\
\hline \multirow{6}{*}{ F. Resultados obtenidos } & 137 & 310 & 3.46 & .676 \\
\hline & 138 & 310 & 3.45 & .650 \\
\hline & 139 & 310 & 3.44 & .654 \\
\hline & 140 & 310 & 3.54 & .681 \\
\hline & 141 & 310 & 3.62 & .593 \\
\hline & 142 & 310 & 3.64 & .584 \\
\hline
\end{tabular}

Fuente: Elaboración propia. 
Estas propiedades reflejan que la bondad métrica del instrumento es aceptable y, en consecuencia, se puede considerar válido y fiable para valorar la opinión del profesorado acerca de los proyectos de trabajo colaborativo con TIC en los que participa.

En conclusión, a la luz de los resultados obtenidos en este estudio, puede afirmarse que el cuestionario PROCOLTIC constituye un instrumento de medida válido y fiable, $\mathrm{y}$ puede ser de utilidad para conocer cómo percibe el profesorado estas experiencias.

Por otra parte, si nos detenemos en el análisis de los resultados obtenidos al aplicar el cuestionario, comprobamos una valoración positiva de los docentes sobre los proyectos en base a su experiencia escolar. Es destacable que los docentes consideran que las tecnologías utilizadas en el proyecto facilitan su adecuada realización. Estos resultados están en sintonía con otros estudios que señalan el valor de las tecnologías para reforzar el ABP, promoviendo la autonomía del estudiante, el acceso a diferentes recursos, y un aprendizaje más enfocado a la investigación e interdisciplinario (CASCALES; CARRILLO; REDOND0, 2017; GÁMIZ, 2017).

Además un aspecto importante que diferencia el $\mathrm{ABP}$ de otros enfoques de instrucción es la colaboración (ROGERS, 2014). Los docentes perciben el ABP como un enfoque que puede impulsar la participación de los estudiantes, brindarles la oportunidad de trabajar con otros compañeros, compartir ideas, ayudarse unos a otros, y aprender de los errores de los demás.

Por otro lado, los docentes han destacado que estas experiencias constituyen una oportunidad importante para la formación del profesorado pues le conduce a la necesidad de decidir y actuar ante múltiples y variados dilemas prácticos y teóricos con lo cual se irá renovando y transformando la intervención de los docentes y su conocimiento profesional (POZUELOS, 2007).

Pero a pesar de las numerosas ventajas, el profesorado sigue encontrando algunas dificultades para implementar con eficacia estas experiencias en el aula, relacionadas principalmente con la escasez de recursos materiales y tecnológicos en los centros, lo que dificulta poder llevar a cabo algunas tareas, así como una falta de apoyo del equipo directivo.

En este sentido, es fundamental que los proyectos estén apoyados y liderados por la dirección del centro, porque motiva a los participantes a implicarse en el proyecto y les impulsa a continuar (GARCÍA VALCÁRCEL; BASILOTTA, 2017; MAJÓ; BAQUERÓ, 2014; PALOMO; RUIZ; SÁNCHEZ, 2005).

También es importante establecer buenos canales de comunicación entre todos los miembros de la comunidad, de manera que conozcan los beneficios que la implantación de un proyecto de trabajo colaborativo puede traer consigo, de este modo asumirán un compromiso mayor con el mismo y el posible recelo que tengan al cambio podrá desaparecer de forma progresiva.

Otro inconveniente que han encontrado algunos docentes está relacionado con la falta de tiempo, no solo por parte de ellos para coordinarse con otros docentes, sino por parte de los alumnos para reflexionar conjuntamente sobre el proceso de trabajo realizado. El aprendizaje basado en proyectos requiere tiempo por parte de los alumnos para discutir, reflexionar, y lograr los aprendizajes previstos. Es evidente que las prácticas 
innovadoras necesitan de un tiempo adicional además de la flexibilidad necesaria para su puesta en práctica (BASILOTTA; MARTÍN; GARCÍA VALCÁRCEL, 2017).

Reflexionar sobre estos inconvenientes puede sernos de gran ayuda para definir y comprender cómo actúan, y qué influencia llegan a tener, y así poder abordarlos con la intención de superarlos progresivamente. Los resultados de esta investigación muestran que la opinión del profesorado es clave para mejorar estas propuestas de enseñanza.

Por último, y dado que la información recogida en el cuestionario a docentes puede estar influida por la deseabilidad social, consideramos necesario incluir entre las evidencias de validación alguna comprobación a través de evaluación externa y/o análisis observacional sobre casos.

También quisiéramos apuntar la necesidad de llevar a cabo otros estudios que examinen la percepción y valoración del alumnado sobre su experiencia educativa en el aprendizaje basado en proyectos que amplíen la información disponible hasta el momento, y mejoren el desarrollo de estas experiencias en el aula.

\section{Referencias}

ALBA, Carmen; CARBALL0, Rafael. Viabilidad de las propuestas metodológicas del crédito europeo por parte del profesorado de las Universidades Españolas, vinculadas a la utilización de las TIC en la docencia y la investigación. Revista de Educación, Madrid, n. 337, p. 71-97, 2005.

ANDREU, María Angeles. Cooperative or collaborative learning: Is there a difference in university students' perceptions? Revista Complutense de Educación, Madrid, v. 27, n. 3, p. 1041-1060, 2016.

ARNAL, Justo; DEL RINCÓN, Delio; LATORRE, Antonio. Investigación educativa: fundamentos y metodologías. Barcelona: Labor, 1992.

BASILOTTA, Verónica; MARTÍN, Marta; GARCÍA-VALCÁRCEL, Ana. Project-Based Learning (PBL) through the incorporation of digital technologies: an evaluation based on the experience of serving teachers. Computers in Human Behavior, Quebec, v. 68, p. 501-512, 2017.

BERNAL, Rosa María. Revisión conceptual y posibilidades educativas de la Web 2.0. Murcia: Universidad de Murcia, 2009. 265 p. Tesis (Doctorado) - Facultad de Educación, Universidad de Murcia, Murcia, 2009. BERTELLE, Adriana; ITURRALDE, Cristina; ROCHA, Adriana. Análisis de la práctica de un docente de ciencias naturales. Revista Iberoamericana de Educación, Madrid, v. 37, n. 4, p. 1-14, 2003.

BLANCHARD, Mercedes. Transformando la sociedad desde las aulas: metodología de aprendizaje por proyectos para la innovación educativa en el Salvador. Madrid: Narcea, 2014.

BRUNDIERS, Katja; WIEK, Arnim. Do we teach what we preach? An international comparative appraisal of problem and project-based learning courses in sustainability. Sustainability, Basilea, v. 5, n. 4, p. 17251746, 2013.

CABERO, Julio; LLORENTE, María del Carmen; MORALES, Juan Antonio. Evaluación del desempeño docente en la formación virtual: ideas para la configuración de un modelo. Revista Iberoamericana de Educación a Distancia, Madrid, v. 21, n. 1, p. 261-279, 2018. 
CASCALES, Antonia; CARRILLO, María Encarnación; REDONDO, Ana María. ABP y tecnología en educación infantil. Pixel-Bit, Sevilla, n. 50, p. 201-209, 2017.

CONDLIFFE, Barbara et al. Project-Based Learning: a literature review. New York: Oaklandd: MDRC, 2017. Disponible en: <https://s3-us-west-1.amazonaws.com/ler/MDRC+PBL+Literature+Review.pdf 2017>. Acceso en: 10 en. 2018.

CORBETTA, Piergiorgio. Metodologías y técnicas de investigación social. Madrid: MC Graw Hill, 2010.

CYPRIAN, Thomas. Teacher self-efficacy in a Project Based Learning (PBL) classroom. Condado de Arlington: [s. n.], 2014. Disponible en: <https://uta-ir.tdl.org/uta-ir/bitstream/handle/10106/24730/CyprianJr_ uta_2502D_12825.pdf?sequence=1 2014>.Acceso en: 15 en. 2018.

DEL RINCÓN, Delio et al. Técnicas de investigación en ciencias sociales. Madrid: Dykinson, 1995.

DU, Xiaomei; HAN, Jie. A literature review on the definition and process of Project-Based Learning and other relative studies. Creative Education, South Croydon, v. 7, p. 1079-1083, 2016.

ENGLISH, Mary. The role of newly prepared PBL teachers' motivational beliefs and perceptions of school conditions in their Project Based Learning implementation. EEUU: ProQuest LLC, 2013.

ERTMER, Peggy; SIMONS, Krista. Jumping the PBL implementation hurdle: supporting the efforts of K-12 teachers. Interdisciplinary Journal of Problem-Based Learning, West Lafayette, v. 1, n. 1, p. 40-54, 2006.

FOGLEMAN, Jay; MCNEILL, Katherine; KRAJCIK, Joseph. Examining the effect of teachers' adaptations of a middle school science inquiry-oriented curriculum unit on student learning. Journal of Research in Science Teaching, Champaign, v. 48, n. 2, p. 149-169, 2011.

GÁMIZ, Vanesa. ICT-based active methodologies. Procedia, Amsterdan, v. 237, p. 606-612, 2017.

GARCÍA, Benilde; LORED0, Javier; CARRANZA, Guadalupe. Análisis de la práctica educativa de los docentes: pensamiento, interacción y reflexión. Redie, Mexicali, v. 10, n. esp., p. 1-15, 2008.

GARCÍA, Esther; CABERO, Julio. Diseño y validación de un cuestionario dirigido a describir la evaluación en procesos de educación a distancia. Edutec, Palma de Mallorca, n. 35, p. 1-26, 2011.

GARCÍA-VALCÁRCEL, Ana; BASILOTTA, Verónica. Aprendizaje Basado en Proyectos (ABP): evalucación desde la perspectiva de alumnos de educación primaria. Revista de Investigación Educativa, Murcia, v. 35, n. 1, p. 113-131, 2017.

HABOK, Anita; NAGY, Judith. In-service teachers' perceptions of Project-Based Learning. SpringerPlus, Heidelberg, v. 5, n. 83, p. 1-14, 2016.

HALVORSEN, Anne-Lise et al. Narrowing the achievement gap in second-grade social studies and content area literacy: the promise of a project-based approach. East Lansing: Michigan State University, Education Policy Center, 2012. 
HARRIS, Christopher et al. Curriculum materials make a difference for next generation science learning: results from year 1 of a randomized controlled trial. Menlo Park: SRI International, 2014.

INTYKBEKOV, Aidyn. Teacher perceptions of project-based learning in a Kazakh-Turkish Lyceum in the northern part of Kazakhstan. Kazajistán: Nazarbayev University, 2017. 88 p. Tesina (Máster of Science in Education) - Nazarbayev University, Kazajistán, 2017.

JOHNSON, David; JOHNSON, Frank. Joining together: group theory and group skills. Boston: Allyn \& Bacon, 2009.

LACUEVA, Aurora. La educación por proyectos abre caminos hacia una escuela más viva y profunda. Red, Ciudad de México, n. 3, p. 68-77, 2016.

LACUEVA, Aurora. Proyectos de aprendizaje en el liceo: hablan 8 docentes. Paradigma,Tegucigalpa, v. 36, n. 1, p. 110-160, 2015.

MADOYAN, Lusine. Authenticity and teacher's role in Project Based Learning. Armenian Folia Anglistika, Ereván, v. 2, n.16, p. 109-114, 2016.

MAJÓ, Francesca; BAQUERÓ, Montserrat. 8 ideas clave: Ios proyectos interdisciplinarios. Barcelona: Graó, 2014.

MCMILLAN, James; SCHUMACHER, Sally. Investigación educativa: una introducción conceptual. Madrid: Pearson, 2005.

MORALES, Pedro. Medición de actitudes en psicología y educación: construcción de escalas y problemas metodológicos. Madrid: Universidad Pontificia de Comillas, 2006.

MORALES, Pedro; UROSA, Belén; BLANCO, Ángeles. Construcción de escalas de actitud tipo Likert: una guía práctica. Madrid: Cuadernos de Estadística, 2003.

OJEA, Manuel. Los proyectos de trabajo: una alternativa educativa como modelo de atención a la diversidad. Revista Española de Pedagogía, Madrid, n. 215, p. 137-152, 2000.

OLMOS, Susana et al. Análisis psicométrico de una escala de percepción sobre la utilidad de moodle en la universidad. Revista Electrónica de Investigación y Evaluación Educativa, Valencia, v. 20, n. 2, p. 1-19, 2014.

ORGANISTA, Javier et al. Desarrollo de un cuestionario para estimar las habilidades digitales de estudiantes universitarios. Revista Complutense de Educación, Madrid, v. 28, n. 1, p. 325-343, 2017.

PALOMO, Rafael; RUIZ, Julio; SÁNCHEZ, José. Las TIC como agentes de innovación educativa. Junta de Andalucía: Consejería de Educación, 2005.

PARKER, Walter et al. Rethinking advanced high school coursework: tackling the depth/breadth tension in the AP US government and politics course. Journal of Curriculum Studies, London, v. 43, n. 4, p. 533-559, 2011.

POZUELOS, Francisco. Trabajo por proyectos: descripción, investigación y experiencias. Morón: MCEP, 2007. 
ROGERS, Tanya. Overcoming implementation challenges with problem and project based learning in advanced technological education programs within community colleges. Boston: Northeastern University, 2014. 121 p. Tesis (Doctorado en Educación) - Northeastern University, Boston, 2014.

RUEDA, Mario. Los retos de la evaluación docente en la universidad. Publicaciones, Granada, v. 48, n. 1, p. 171-192, 2018.

SALCINES, Irina; GONZÁLEZ, Natalia. Diseño y validación del cuestionario smartphone y universidad: visión del profesorado (SUOL). Revista Complutense de Educación, Madrid, v. 27, n. 2, p. 603-632, 2016.

THAMARASSERI, Ismail; Convergence of Information and Communication Technology (ICT) tools in Project Based Learning (PBL). I-manager's Journal on School Educational Technology (JSCH), New York, v. 10, n. 1, p. 1-7, 2014.

THOMAS, John. A review of research on project-based learning. The San Rafael: Autodesk Foundation, 2000. Disponible en: <http://www.bobpearlman.org/BestPractices/PBL_Research.pdf 2000>. Acceso en: 20 en. 2018.

VEGA, Vanessa. Investigaciones sobre el ABP. Revista Aula, Barcelona, n. 247, p. 53-59, 2015.

ZABALA, Antoni. La práctica educativa, cómo enseñar. Barcelona: Grao, 2002.

Recibido en: 09.09.2018

Modificaciones en: 09.11.2018

Aprobado en: 28.11.2018

Verónica Basilotta Gómez-Pablos es profesora en el Departamento de Educación de la Universidad a Distancia de Madrid. Doctora en Ciencias de la Educación por la Universidad de Salamanca. Miembro del Grupo de Investigación GITE-USAL.

Ana García-Valcárcel Muñoz-Repiso es catedrática de Tecnología Educativa en la Facultad de Educación de la Universidad de Salamanca. Directora del Máster Oficial Las TIC en Educación, y del Grupo de Investigación GITE-USAL. 
Anexo 1- PROCOLTIC. Cuestionario definitivo

\begin{tabular}{|c|c|c|c|c|c|c|}
\hline $\mathrm{N}$ & Ítem & & & tua & & \\
\hline 1 & $\begin{array}{l}\text { Las características del centro/aula son apropiadas/ } \\
\text { adecuadas para el desarrollo de proyectos colaborativos con } \\
\text { TIC (instalaciones, espacios, etc.) }\end{array}$ & 0 & 1 & 2 & 3 & 4 \\
\hline 2 & $\begin{array}{l}\text { El centro dispone de equipos tecnológicos adecuados } \\
\text { para su uso pedagógico (ordenadores, TV, DVD, escáner, } \\
\text { impresora, pizarra digital, máquina fotográfica, vídeo, equipo } \\
\text { de sonido) }\end{array}$ & 0 & 1 & 2 & 3 & 4 \\
\hline 3 & $\begin{array}{l}\text { El centro dispone de una velocidad de conexión a Internet } \\
\text { adecuada. }\end{array}$ & 0 & 1 & 2 & 3 & 4 \\
\hline 4 & $\begin{array}{l}\text { Existe una coordinación adecuada entre el profesorado } \\
\text { implicado en el desarrollo del proyecto. }\end{array}$ & 0 & 1 & 2 & 3 & 4 \\
\hline 5 & $\begin{array}{l}\text { La actitud del profesorado hacia el desarrollo de actividades } \\
\text { colaborativas con TIC es positiva. }\end{array}$ & 0 & 1 & 2 & 3 & 4 \\
\hline 6 & $\begin{array}{l}\text { El equipo directivo del centro apoya e impulsa el desarrollo } \\
\text { del proyecto. }\end{array}$ & 0 & 1 & 2 & 3 & 4 \\
\hline 7 & $\begin{array}{l}\text { El proyecto responde a un diseño previamente planificado y } \\
\text { consensuado. }\end{array}$ & 0 & 1 & 2 & 3 & 4 \\
\hline 8 & Los objetivos de aprendizaje están claramente definidos. & 0 & 1 & 2 & 3 & 4 \\
\hline 9 & $\begin{array}{l}\text { Los contenidos del proyecto son relevantes y se ajustan al } \\
\text { currículo oficial. }\end{array}$ & 0 & 1 & 2 & 3 & 4 \\
\hline 10 & $\begin{array}{l}\text { Las actividades son motivadoras y despiertan la curiosidad } \\
\text { del alumnado. }\end{array}$ & 0 & 1 & 2 & 3 & 4 \\
\hline 11 & $\begin{array}{l}\text { La distribución, estructura y organización de las tareas es } \\
\text { adecuada. }\end{array}$ & 0 & 1 & 2 & 3 & 4 \\
\hline 12 & $\begin{array}{l}\text { Las tecnologías utilizadas en el proyecto facilitan su } \\
\text { adecuada realización. }\end{array}$ & 0 & 1 & 2 & 3 & 4 \\
\hline 13 & $\begin{array}{l}\text { El proyecto permite una mayor interacción del docente con } \\
\text { su alumnado. }\end{array}$ & 0 & 1 & 2 & 3 & 4 \\
\hline 14 & $\begin{array}{l}\text { El proyecto promueve la implicación activa del alumnado en } \\
\text { su proceso de aprendizaje. }\end{array}$ & 0 & 1 & 2 & 3 & 4 \\
\hline 15 & $\begin{array}{l}\text { Ha participado (participa) activamente en la planificación y } \\
\text { diseño del proyecto. }\end{array}$ & 0 & 1 & 2 & 3 & 4 \\
\hline 16 & $\begin{array}{l}\text { Ha participado (participa) activamente en el desarrollo del } \\
\text { proyecto. }\end{array}$ & 0 & 1 & 2 & 3 & 4 \\
\hline 17 & $\begin{array}{l}\text { Ha tenido (tiene) una actitud favorable durante el desarrollo } \\
\text { del proyecto. }\end{array}$ & 0 & 1 & 2 & 3 & 4 \\
\hline 18 & $\begin{array}{l}\text { Se ha sentido (se siente) cómodo empleando las herramientas } \\
\text { tecnológicas utilizadas en el proyecto. }\end{array}$ & 0 & 1 & 2 & 3 & 4 \\
\hline 19 & $\begin{array}{l}\text { Ha animado (anima) al alumnado más reticente a realizar las } \\
\text { actividades del proyecto. }\end{array}$ & 0 & 1 & 2 & 3 & 4 \\
\hline 20 & $\begin{array}{l}\text { Ha llevado (lleva) a cabo un seguimiento y registro del trabajo } \\
\text { realizado por el alumnado durante el proyecto. }\end{array}$ & 0 & 1 & 2 & 3 & 4 \\
\hline 21 & $\begin{array}{l}\text { Ha elaborado sus propios materiales didácticos para } \\
\text { adaptarlos a su alumnado (juegos, presentaciones, vídeos, } \\
\text { actividades, etc.). }\end{array}$ & 0 & 1 & 2 & 3 & 4 \\
\hline 22 & $\begin{array}{l}\text { Ha utilizado diferentes herramientas que facilitan la } \\
\text { comunicación (redes sociales, mensajería instantánea, } \\
\text { videoconferencia...) }\end{array}$ & 0 & 1 & 2 & 3 & 4 \\
\hline
\end{tabular}




\begin{tabular}{|c|c|c|c|c|c|c|}
\hline 23 & $\begin{array}{l}\text { Ha utilizado diferentes herramientas de la Web } 2.0 \text { que } \\
\text { facilitan la creación y publicación de contenidos (blog, wiki, } \\
\text { webquest, podcast, ofimática colaborativa: docs...) }\end{array}$ & 0 & 1 & 2 & 3 & 4 \\
\hline 24 & $\begin{array}{l}\text { Ha utilizado diferentes herramientas de la Web } 2.0 \text { que } \\
\text { facilitan la gestión de información (dropbox, RSS, diigo, } \\
\text { delicious, buscadores, escritorios especializados...) }\end{array}$ & 0 & 1 & 2 & 3 & 4 \\
\hline 25 & $\begin{array}{l}\text { Ha utilizado diferentes estrategias o instrumentos para } \\
\text { registrar el desempeño y los aprendizajes adquiridos por el } \\
\text { alumnado (ejercicios prácticos, diario de reflexión, portfolio, } \\
\text { rúbrica, etc.). }\end{array}$ & 0 & 1 & 2 & 3 & 4 \\
\hline 26 & $\begin{array}{l}\text { El alumnado ha asumido responsabilidades en las tareas } \\
\text { asignadas. }\end{array}$ & 0 & 1 & 2 & 3 & 4 \\
\hline 27 & $\begin{array}{l}\text { El alumnado ha asumido roles complementarios para el } \\
\text { desempeño de las tareas. }\end{array}$ & 0 & 1 & 2 & 3 & 4 \\
\hline 28 & $\begin{array}{l}\text { El alumnado ha sido más autónomo en su aprendizaje } \\
\text { mediado con TIC. }\end{array}$ & 0 & 1 & 2 & 3 & 4 \\
\hline 29 & $\begin{array}{l}\text { El alumnado ha tenido tiempo para reflexionar conjuntamente } \\
\text { sobre el proceso de trabajo realizado. }\end{array}$ & 0 & 1 & 2 & 3 & 4 \\
\hline 30 & $\begin{array}{l}\text { El alumnado ha tenido suficientes oportunidades para } \\
\text { interactuar y colaborar con sus compañeros. }\end{array}$ & 0 & 1 & 2 & 3 & 4 \\
\hline 31 & $\begin{array}{l}\text { El alumnado se ha sentido apoyado por sus compañeros en } \\
\text { el desarrollo del proyecto, le han proporcionado ayuda, ánimo } \\
\text { y refuerzo. }\end{array}$ & 0 & 1 & 2 & 3 & 4 \\
\hline 32 & $\begin{array}{l}\text { El alumnado ha ayudado a los compañeros con dificultades } \\
\text { para realizar las tareas. }\end{array}$ & 0 & 1 & 2 & 3 & 4 \\
\hline 33 & $\begin{array}{l}\text { El alumnado ha usado adecuadamente las tecnologías en las } \\
\text { actividades del proyecto. }\end{array}$ & 0 & 1 & 2 & 3 & 4 \\
\hline 34 & $\begin{array}{l}\text { El alumnado ha participado activamente en las actividades } \\
\text { del proyecto. }\end{array}$ & 0 & 1 & 2 & 3 & 4 \\
\hline 35 & $\begin{array}{l}\text { La actitud del alumnado ha sido óptima para el desempeño/ } \\
\text { desarrollo del proyecto. }\end{array}$ & 0 & 1 & 2 & 3 & 4 \\
\hline 36 & $\begin{array}{l}\text { La actitud del alumnado ha fomentado un clima de respeto y } \\
\text { confianza en el aula. }\end{array}$ & 0 & 1 & 2 & 3 & 4 \\
\hline 37 & $\begin{array}{l}\text { El proyecto le ha permitido aprender-adquirir competencias } \\
\text { tecnológicas para la docencia. }\end{array}$ & 0 & 1 & 2 & 3 & 4 \\
\hline 38 & $\begin{array}{l}\text { El proyecto le ha permitido aprender-adquirir competencias } \\
\text { pedagógicas para la docencia (enseñar, etc.) }\end{array}$ & 0 & 1 & 2 & 3 & 4 \\
\hline 39 & $\begin{array}{l}\text { El proyecto le ha permitido aprender-adquirir competencias } \\
\text { sociales (compañerismo, trabajo en equipo, respeto, etc.) }\end{array}$ & 0 & 1 & 2 & 3 & 4 \\
\hline 40 & $\begin{array}{l}\text { El proyecto promueve la participación activa del profesorado } \\
\text { en redes y comunidades de aprendizaje para compartir } \\
\text { experiencias con sus compañeros docentes. }\end{array}$ & 0 & 1 & 2 & 3 & 4 \\
\hline 41 & $\begin{array}{l}\text { El proyecto es una buena oportunidad para la formación del } \\
\text { profesorado. }\end{array}$ & 0 & 1 & 2 & 3 & 4 \\
\hline 42 & $\begin{array}{l}\text { El proyecto es una oportunidad para un cambio en la cultura } \\
\text { de los centros. }\end{array}$ & 0 & 1 & 2 & 3 & 4 \\
\hline
\end{tabular}

